\title{
Glass Transition in Suspensions of Charged Rods: Structural Arrest and Texture Dynamics
}

\author{
K. Kang and J. K. G. Dhont \\ Forschungszentrum Jülich, Institute of Complex Systems, ICS-3, D-52425 Jülich, Germany
}

(Received 29 August 2012; published 2 January 2013)

\begin{abstract}
We report on the observation of a glass transition in suspensions of very long and thin, highly charged colloidal rods (fd-virus particles). Structural particle arrest is found to occur at a low ionic strength due to caging of the charged rods in the potential setup by their neighbors through long-ranged electrostatic interactions. The relaxation time of density fluctuations as probed by dynamic light scattering is found to diverge within a small concentration range. The rod concentration where structural particle arrest occurs is well within the full chiral-nematic state, far beyond the two-phase isotropic-nematic coexistence region. The morphology of the suspensions thus consists of nematic domains with various orientations. We quantify the dynamics of the resulting texture with image-time correlation spectroscopy. Interestingly, the decay times of image correlation functions are found to diverge in a discontinuous fashion at the same concentration of charged rods where structural particle arrest is observed. At the glass-transition concentration, we thus find both structural arrest and freezing of the texture dynamics.
\end{abstract}

DOI: 10.1103/PhysRevLett.110.015901

PACS numbers: 66.30.hh, 64.70.pv, 64.70.pp

Glass transitions have been observed for many different types of systems, ranging from polymeric systems and simple liquids to colloids. The common feature of glass forming systems is that particle dynamics becomes very slow on approach of the glass-transition point, so that structural relaxation times exceed experimental time scales. The nature of the glass transition is still under debate and is sometimes stated as one of the most outstanding open problems in physics [1-3]. The probably most simple systems that form a glass are colloidal suspensions of hard spheres, which have been extensively investigated [4-6]. Other types of repulsive colloidal systems consisting of spherical colloids have been studied subsequently, such as repulsive microgel particles [7], charged colloids [8], starlike colloids [9-12], and hardsphere colloid-polymer mixtures, where depletion attractions between the colloids induced by the polymers also play an important role [13]. Some overview papers that are relevant for colloids are Refs. [1,14-18], as well as the book by Donth [19].

An intuitive view of the mechanism leading to structural arrest of colloidal suspensions can be formulated in terms of a "cage" around each individual particle that is formed by its neighbors. For short times a colloidal particle diffuses within the cage (the so-called $\beta$ process) while longtime diffusion relates to rare cage escape (the $\alpha$ process). It is the latter diffusion process that becomes arbitrarily slow at sufficiently high packing fractions.

Less work has been done on the glass transition of repulsive nonspherical colloids. There is an experimental study [20] on rodlike colloids with large aspect ratios, where a glass transition is reported to occur within the nematic state. A glass transition was also recently found for slightly nonspherical particles, with aspect ratios too small to exhibit an isotropic-nematic phase transition [21].
Simulations on the packing characteristics of such short rods are reported in Refs. [22,23]. Structural arrest has also been found in quasi-2D systems of ellipsoidal rods by means of microscopy [24]. In this Letter we report on a glass transition in suspensions of very long and thin, highly charged colloidal rods (fd-virus particles) with long-ranged repulsive electrostatic interactions. At the glass-transition concentration we find (i) structural arrest, as observed by means of dynamic light scattering, at a concentration far above the isotropic-nematic two-phase coexistence region, and (ii) freezing of the texture dynamics, as probed with image time-correlation spectroscopy. Within the intuitive view mentioned above, the cages are now formed through long-ranged electrostatic repulsive interactions.

There are recent extensions of the classic mode coupling theory for spherical colloids as pioneered by Götze and co-workers $[25,26]$ to ellipsoidal colloids with hard-core interactions [27-29]. The latter two references, which also include hopping, relate to very short rods which do not exhibit an isotropic-nematic phase transition, and are therefore not applicable to the present system of very long and thin rods. In Ref. [27], and also in simulations [30], an intervening isotropic-nematic phase transition is predicted before particle arrest occurs, which is also seen in our experiments.

The systems we use are suspensions of fd-virus particles, which consist of a DNA strand covered with about 2700 coat proteins that carry a total bare charge of 10000 elementary charges. The length of a fd-virus particle is $880 \mathrm{~nm}$, the core thickness is $6.8 \mathrm{~nm}$, and the persistence length is $2500 \mathrm{~nm}$. In the past decades, fd-virus particles have been used as a model system for very long and thin, stiff and highly charged colloidal rods. Suspensions of fd-virus particles have been shown to form many different liquid-crystalline equilibrium phases at a high ionic 
strengths (between about 1 and $200 \mathrm{mM}$ ) [31-34]. The ionic strength in the present experiments is far lower, $0.16 \mathrm{mM}$, corresponding to a relatively large Debye length of $27 \mathrm{~nm}$. The lower isotropic-nematic binodal fd concentration for this low buffer concentration is $1.5 \pm$ $0.2 \mathrm{mg} / \mathrm{ml}$ and the upper binodal concentration is $3.4 \pm 0.5 \mathrm{mg} / \mathrm{ml}$. The resulting long-ranged electrostatic interactions lead to caging beyond the glass-transition concentration, where each particle is trapped within the potential landscape set up by the neighboring particles.

Experiments are performed with the use of cylindrical cuvettes with a diameter of $20 \mathrm{~mm}$ and a height of $1 \mathrm{~mm}$. The fd-virus particles are slightly birefringent, so that local orientational ordering can be visualized by observing the cuvette between crossed polarizers. Images are taken with an AxioCam Color A12-312 CCD camera, with a chip of $432 \times 432$ pixels. Measurements of correlation functions, and recording of images, are started after a certain time at which the cuvette was filled with the suspension. This time will be referred to as the "waiting time."

Shortly after filling the cuvette, very large nematic domains are present due to initial shear alignment, as can be seen in Fig. 1(a) for a fd concentration of $4.0 \mathrm{mg} / \mathrm{ml}$. The top-left CCD camera image in Fig. 1(a) shows the entire cuvette right after filling it with the fd suspension, the top-right image after a waiting time $t_{w}$ of $5 \mathrm{~h}$. Equilibration of the chiral-nematic texture is typically achieved after $50-100 \mathrm{~h}$, as can be seen from the lower row of three images in Fig. 1(a). These images cover an area of $7 \times 7 \mathrm{~mm}^{2}$, corresponding to the yellow square in the top-right image in Fig. 1(a). Equilibration times and the texture dynamics after equilibration are quantified by means of image time-correlation spectroscopy [35,36]. From time traces of the transmitted intensity as recorded by the CCD camera, we compute intensity correlation functions, which we will refer to as "image timecorrelation functions" $C_{V}(t)$ (the index " $V$ " stands for video). Each single image in a time trace consists of $170 \times$ 170 pixels. It is important to note that the time $t$ in the time image time-correlation function $C_{V}(t)$ is taken from the start of the measurement of the correlation function; that is, $t$ is zero at the time the measurement is started, having waited for a time $t_{w}$ after filling the cuvette. The "initial decay rate" of the correlation functions that we will use to characterize the texture dynamics therefore relates to the time dependence of the correlation functions for small times $t$. Typical image-time correlation functions are shown in Fig. 1(b) for various waiting times $t_{w}$. Shortly after filling the cuvette, the extended initially shear-aligned nematic domains break down into smaller domains, leading to a relatively fast decay of the image correlation function. After about $50-100 \mathrm{~h}$, the correlation functions become independent of the waiting time. Beyond those waiting times the nematic texture reached equilibrium. Note that the relaxation times of image time-correlation
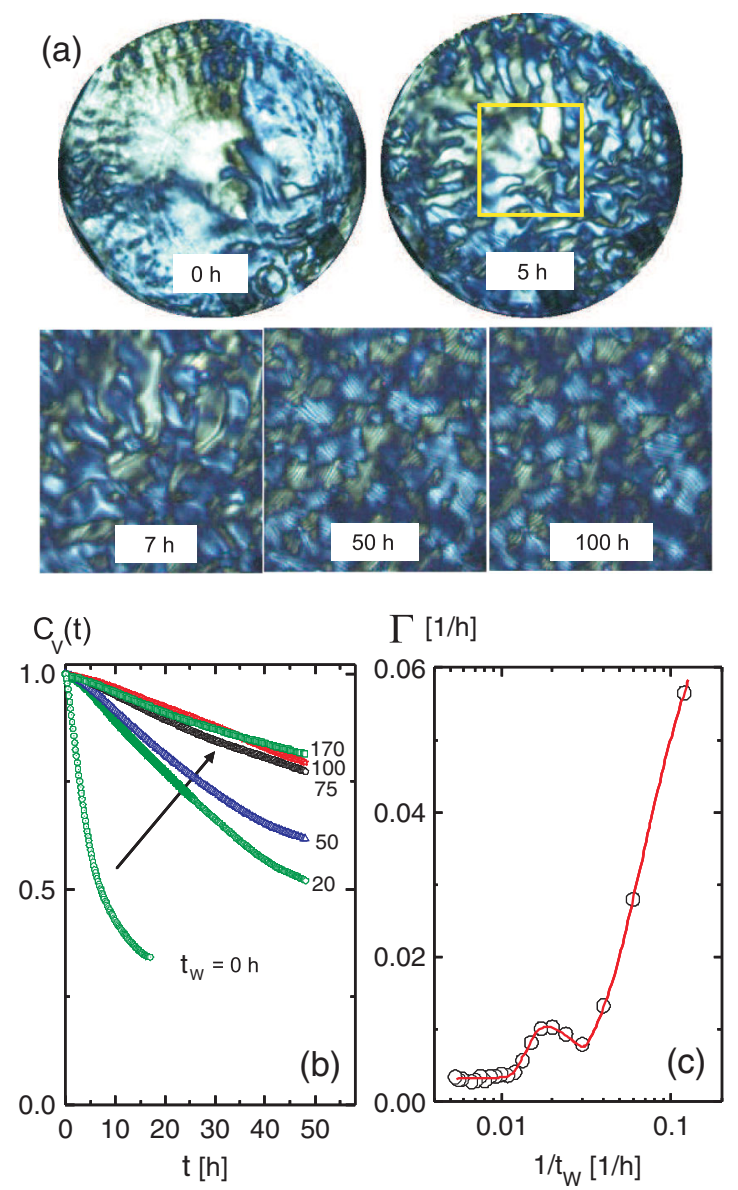

FIG. 1 (color). (a) Depolarized CCD images for a fd concentration of $4.0 \mathrm{mg} / \mathrm{ml}$. The top row shows images of the entire cuvette, with a diameter of $20 \mathrm{~mm}$, at two waiting times $t_{w}$ as indicated. The three images below cover $7 \times 7 \mathrm{~mm}^{2}$ areas (see the yellow indicated square in the top-right image), for longer waiting times. These types of images are used to construct image time-correlation functions. (b) Image time-correlation functions for different waiting times (given in hours), as indicated in the figure. (c) The initial decay rate $\Gamma$ as a function of the inverse waiting time.

functions in the equilibrated state are of the order of a few hundreds of hours, so that it is only feasible to perform systematic measurements on the initial decay rate $\Gamma$. The initial decay rate is shown in Fig. 1(c) as a function of the inverse waiting time. The value of $\Gamma$ after equilibration is taken to characterize the dynamics of the texture. The characteristic behavior shown in Fig. 1 for a fd concentration of $4.0 \mathrm{mg} / \mathrm{ml}$ is essentially unchanged up to $11.1 \mathrm{mg} / \mathrm{ml}$. At a slightly larger concentration of $12.3 \mathrm{mg} / \mathrm{ml}$, the behavior of the texture dynamics changes drastically. The initially shear-aligned morphology does not break down to smaller domains anymore, as can be seen from the images in Fig. 2(a) (these images show the entire cuvette). The initial morphology is frozen on time scales of at least $50-100 \mathrm{~h}$. The corresponding image correlation functions are now unchanged right after filling 

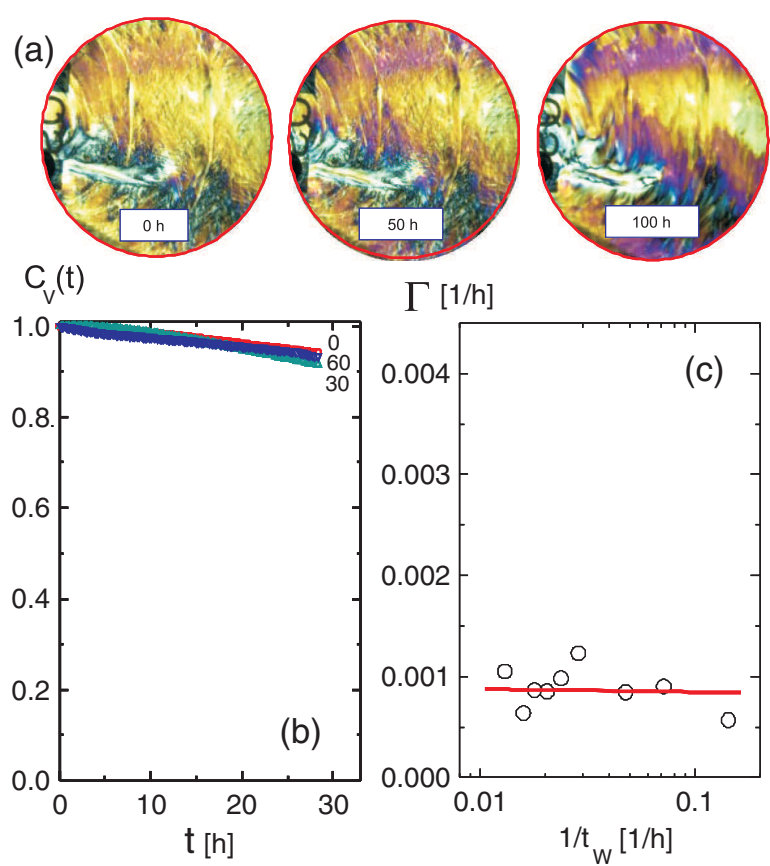

$\Gamma[1 / h]$

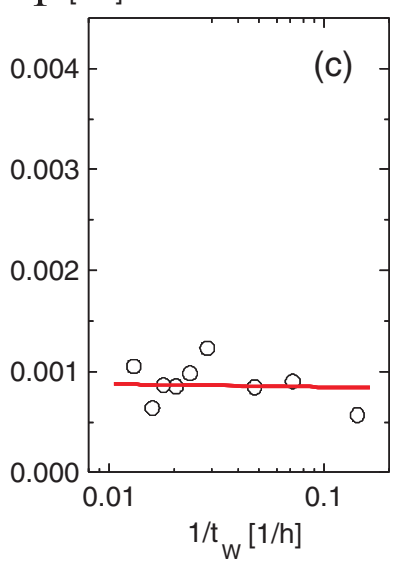

FIG. 2 (color). Same as in Fig. 1, but now for a fd concentration of $13.6 \mathrm{mg} / \mathrm{ml}$. In (a) images of the entire cuvette are given, (b) the image time-correlation functions for different waiting times (given in hours), and (c) plot of the initial relaxation rate as a function of the inverse waiting time.

the cuvette, as can be seen in Fig. 2(b). The initial decay rates are now constant, independent of the waiting time, as shown in Fig. 2(c). The small but still finite initial decay rate is due to slow release of the stresses that resulted from shear alignment.

The plot in Fig. 3 shows the initial decay rates of image time-correlation functions for a series of concentrations. Clearly, the decay rates for the equilibrated morphologies below about $11.7 \pm 0.6 \mathrm{mg} / \mathrm{ml}$ are independent of concentration. There is a discontinuous jump of the initial relaxation rate to a much smaller value above this concentration. The nematic texture thus "freezes" at concentrations larger than $11.7 \pm 0.6 \mathrm{mg} / \mathrm{ml}$. The texture-freezing concentration lies within the gray area in Fig. 3. The images of the entire cuvette in Fig. 3 on the right illustrate the very different behavior of the texture dynamics just below and above the texture-freezing transition, within a waiting time of $40 \mathrm{~h}$. The two images at the top show the texture at the concentration $11.1 \mathrm{mg} / \mathrm{ml}$, just below the texture-freezing transition concentration, and the bottom two images are for $12.3 \mathrm{mg} / \mathrm{ml}$, just above the glasstransition concentration. The left-hand images are taken right after filling the cuvette, while the images on the right are taken after a waiting time of $40 \mathrm{~h}$.

To probe the microscopic dynamics of the charged colloidal fd rods, we used dynamic light scattering (DLS), with an ALV-5000/EPP multiple tau digital real time correlator. The wavelength of the $\mathrm{He}-\mathrm{Ne}$ laser is

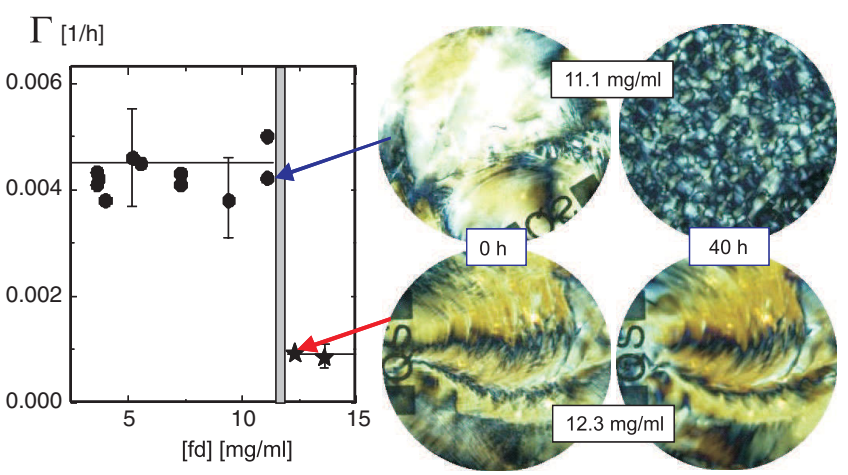

FIG. 3 (color). Initial image time-correlation decay rates as a function of fd concentration. The top two images on the right are for the fd concentration of $11.1 \mathrm{mg} / \mathrm{ml}$ just below the texturefreezing concentration, and the bottom two images for a concentration of $12.3 \mathrm{mg} / \mathrm{ml}$ just above the glass transition. The left-hand images are taken right after filling the cuvette, the right-hand images after a waiting time of $40 \mathrm{~h}$.

$633 \mathrm{~nm}$ (JDS Uniphase Model 1145P), and the scattering angle is chosen as $16^{\circ}$. We tested the stability of the setup by recording DLS correlation functions from a scratched glass plate. Correlation functions are found to be reliable over a time range of $800 \mathrm{~s}$. The laser wavelength and scattering angle correspond to a length scale close to $1 \mu \mathrm{m}$, so that displacements of rods are probed over distances which are comparable with their length. Below the texture-freezing transition concentration, DLS correlation functions are collected after waiting for $100 \mathrm{~h}$, the waiting time at which the nematic texture equilibrated. Typical recording times for time-averaged correlation functions vary in the range of 3-15 h. During this time the texture is essentially unchanged. The ensemble averaged normalized DLS correlation function $\hat{g}_{E}$ is calculated by a brute-force averaging of time-averaged correlation functions. Averages over typically $N=5-7$ correlation functions, taken at different positions in the sample, turned out to be sufficient to obtain reliable ensemble-averaged correlation functions. For the small length scale of $1 \mu \mathrm{m}$ that we probe, orientational dynamics is not seen in correlation functions. The decay time is entirely determined by translational motion. For concentrations above the texturefreezing concentration, DLS correlation functions are similarly obtained, but now also for shorter waiting times, since equilibration of the texture no longer occurs. The correlation functions for short and long waiting times are found to be identical. DLS correlation functions for various fd concentrations are shown in Fig. 4(a). As can be seen, there is a sharp transition, within a small concentration range, to a nondecaying correlation function (on a time scale of $\sim 1 \mathrm{~h}$ ). The concentration at which the relaxation time diverges is the glass-transition concentration where structural particle arrest occurs. The solid lines are fits to a stretched exponential $\exp \left\{-(t / \tau)^{\beta}\right\}$, which is the form of correlation functions as predicted by mode-coupling 

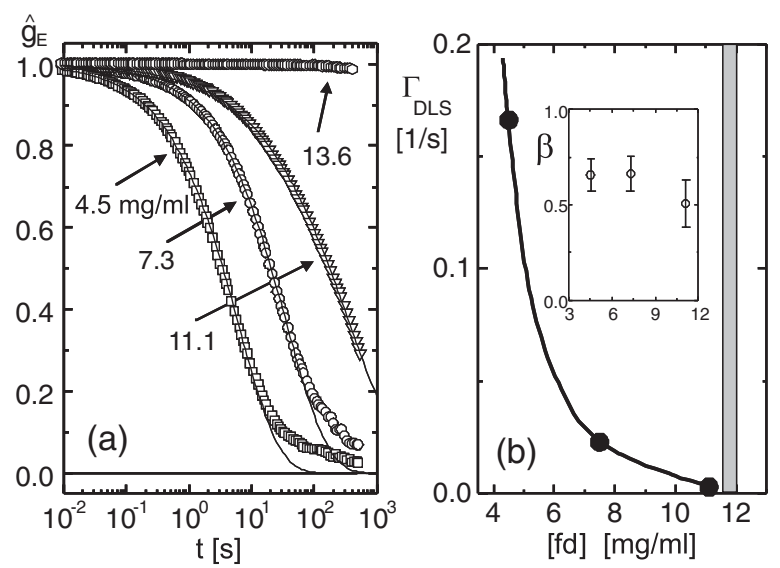

FIG. 4. (a) Ensemble averaged DLS correlation functions for various fd concentrations. Solid lines are fits to a stretched exponential function. (b) The decay rate $\Gamma_{\mathrm{DLS}}=1 / \tau$ as a function of fd concentration. The gray region indicates the region where structural arrest occurs. The inset shows the stretching exponential $\beta$ as a function of fd concentration.

theory. The slow decay for somewhat longer times seen in Fig. 4(a) might be due to the slight rotation of domains during the measuring time of correlation functions, or might be connected to slow elastic modes. These longer times are not included in the fit. The time constant $\tau$ diverges (or more precisely, is much larger than $800 \mathrm{~s}$ ) at a concentration where the texture is also found to freeze (to within $\sim 1 \mathrm{mg} / \mathrm{ml}$ ). The DLS decay rate $\Gamma_{\mathrm{DLS}}=1 / \tau$ is plotted as a function of fd concentration in Fig. 4(b), where the gray region indicates the location of the texturefreezing concentration. As can be seen from the inset in Fig. 4(b), the stretching factor $\beta$ is slightly concentration dependent and attains a value of $0.50 \pm 0.08$ close to the glass transition.

We have thus shown that very long and thin, charged colloidal rods with long-ranged electrostatic, purely repulsive interactions exhibit a glass transition, where both structural particle arrest occurs and the domain texture freezes at concentrations well within the full nematic state.

Since the observed structural arrest is due to electrostatic repulsive interactions, we expect that the glass-transition $\mathrm{fd}$ concentration will be lower on lowering the ionic strength, due to the increasing extent of the double layer. We also observed that in the glass the fd suspensions exhibit a yield stress, complying with structural arrest.

[1] L. Berthier and G. Biroli, Rev. Mod. Phys. 83, 587 (2011).

[2] P. G. Debenedetti and F.H. Stillinger, Nature (London) 410, 259 (2001).

[3] C. A. Angell, Science 267, 1924 (1995).

[4] P. N. Pusey and W. Megen, Nature (London) 320, 340 (1986).
[5] W. van Megen and S. M. Underwood, Phys. Rev. E 49, 4206 (1994).

[6] W. van Megen, T.C. Mortensen, S. R. Williams, and J. Muller, Phys. Rev. E 58, 6073 (1998).

[7] E. Bartsch, M. Antonietti, W. Schupp, and H. Sillescu, J. Chem. Phys. 97, 3950 (1992).

[8] C. H. Beck, W. Härtl, and R. Hempelmann, J. Chem. Phys. 111, 8209 (1999).

[9] J. Stellbrink, J. Allgaier, and D. Richter, Phys. Rev. E 56, R3772 (1997).

[10] D. Vlassopoulous, J. Polym. Sci. B 42, 2931 (2004).

[11] C. Mayer, E. Zaccarelli, E. Stiakakis, C. N. Likos, F. Sciortino, A. Munam, M. Gauthier, N. Hadjichristidis, H. Iatrou, P. Tartaglia, H. Löwen, and D. Vlassopoulous, Nat. Mater. 7, 780 (2008).

[12] D. Vlassopoulous and G Fytas, Adv. Polym. Sci. 236, 1 (2000).

[13] W.C. K. Poon, J. Phys. Condens. Matter 14, R859 (2002).

[14] K. Binder and J. D. Reger, Adv. Phys. 41, 547 (1992).

[15] W. van Megen, J. Phys. Condens. Matter 14, 7699 (2002).

[16] C. A. Angell, MRS Bull. 33, 544 (2008).

[17] H. Tanaka, J. Stat. Mech. 12 (2010) P12001.

[18] M. J. Solomon and P.T. Spicer, Soft Matter 6, 1391 (2010).

[19] E. Donth, The Glass Transition: Relaxation Dynamics in Liquids and Disordered Materials (Springer, Berlin, 2001).

[20] A. Wierenga, A. P. Philipse, and H. N. W. Lekkerkerker, Langmuir 14, 55 (1998).

[21] R.C. Kramb, R. Zhang, K.S. Schweizer, and C.F. Zukoski, Phys. Rev. Lett. 105, 055702 (2010).

[22] C.F. Schreck, M. Mailman, B. Chakraborty, and C.S. O'Hern, Phys. Rev. E 85, 061305 (2012).

[23] C.F. Schreck, N. Xu, and C.S. O'Hern, Soft Matter 6, 2960 (2010).

[24] Z. Zheng, F. Wang, and Y. Han, Phys. Rev. Lett. 107, 065702 (2011).

[25] W. Götze and L. Sjögren, Z. Phys. B 65, 415 (1987).

[26] G. Buchalla, U. Dersch, W. Götze, and L. Sjögren, J. Phys. C 21, 4239 (1988).

[27] M. Letz, R. Schilling, and A. Latz, Phys. Rev. E 62, 5173 (2000).

[28] G. Yatsenko and K. S. Schweizer, J. Chem. Phys. 126, 014505 (2007).

[29] G. Yatsenko and K. S. Schweizer, Phys. Rev. E 76, 041506 (2007).

[30] C. De Michele, R. Schilling, and F. Sciortino, Phys. Rev. Lett. 98, 265702 (2007).

[31] Z. Dogic and S. Fraden, Langmuir 16, 7820 (2000).

[32] E. Grelet and S. Fraden, Phys. Rev. Lett. 90, 198302 (2003).

[33] S. Fraden, Phil. Trans. R. Soc. A 359, 997 (2001).

[34] S. Fraden, in Phase Transitions in Colloidal Suspensions of Virus Particles, NATO Advanced Study Institutes, Ser. C, 460 (Kluwer Academic, Dordrecht, 1995), p. 113.

[35] K. Kang and J. K. G. Dhont, Eur. Phys. J. E 30, 333 (2009).

[36] K. Kang, Rev. Sci. Instrum. 82, 053903 (2011). 